\title{
Análise dos determinantes de eficiência educacional do estado do Ceará
}

\author{
Maria Cristina Gramani a
}

\section{Resumo}

O Estado do Ceará vem apresentando melhorias significativas no setor da educação desde 2007, principalmente nos anos iniciais do Ensino Fundamental, onde já superou as metas de avaliação da educação estabelecidas pelo governo. Nesse sentido, este artigo busca identificar os determinantes de eficiência que levaram este Estado a melhorar seu desempenho educacional, considerando além de variáveis ligadas diretamente à educação também variáveis socioeconômicas, que exercem grande influência nesse setor. Os resultados mostram claramente a existência de clusters de municípios considerados benchmarking que geograficamente coincidem com as localizações dos três campi do curso de Medicina da Universidade Federal do Ceará. As variáveis renda, gasto per capita e educação materna se mostraram como determinantes de eficiência dos municípios cearenses, mas a variável relacionada ao acesso a esgoto sanitário se mostra um forte determinante de ineficiência, principalmente para os municípios menos favorecidos.

Palavras-chave: Educação básica. Qualidade de ensino. Eficiência.

\section{Introdução}

No Brasil, embora diversos esforços provenientes de vários setores tanto governamentais como privados estejam levando a uma melhoria da educação, os resultados ainda não atingiram um patamar aceitável. Em 2012, constatou-se que apenas 32,9\% dos alunos brasileiros encontram-se no nível 2 ou acima nas edições do PISA (Programa Internacional de Avaliação de Estudantes), que tem o nível 6 como teto. (Fonte: www.observatoriodopne.org.br).

Diversas melhorias no país vêm surgindo por meio de ações governamentais ou, até mesmo, por meio de ações isoladas de municípios. Nesse sentido, o estudo de boas práticas escolares pode auxiliar na identificação de fatores que impulsionam o

\footnotetext{
a Universidade Estadual de Campinas. Campinas, São Paulo, Brasil.
} 
desempenho acadêmico, incluindo no estudo características socioeconômicas de cada escola ou cada município a ser analisado. De uma forma mais ampla, o estudo das boas práticas escolares é altamente recomendado pelas agências multilaterais - como o Banco Mundial, o Banco Interamericano de Desenvolvimento (BID), a Organização das Nações Unidas para a Educação, a Ciência e a Cultura (Unesco), e o Fundo das Nações Unidas para a Infância (Unicef) - e estimulado pelos governos de diversos países, incluindo o Brasil. (CALDERÓN et al., 2015).

A publicação do Fundo das Nações Unidas para a Infância, em 2010, "Caminhos do direito de aprender", fez uma análise da trajetória de 26 municípios que conquistaram avanços significativos no Índice de Desenvolvimento da Educação Básica (IDEB), entre 2005 e 2007. Esse estudo categorizou os avanços em quatro fatores: fator desencadeador (todas as redes analisadas atribuíram ao resultado do IDEB 2005 parte do seu progresso em 2007), fator catalisador (a gestão foi apontada pelos municípios como ingrediente fundamental para o sucesso das redes), fatores de avanço (formação de professores, práticas pedagógicas e ambiente de aprendizagem) e, finalmente, fatores de base (valorização profissional e boas condições de trabalho, ampliação do tempo na escola e documentos norteadores).

A Capes (Coordenação de Aperfeiçoamento de Pessoal de Nível Superior) que, desde 2007, assumiu a missão na educação básica de induzir, fomentar e valorizar a formação inicial e continuada de profissionais de magistério, vem promovendo ações para a melhoria da educação básica, por exemplo, oferecer matrículas em cursos de formação inicial do Parfor, por meio da Plataforma Freire, oferecer programas de formação continuada qualificada, stricto e lato sensu, aos professores que adquiram nível superior (CLÍMACO, 2012).

Além disso, ações individuais promovidas por Estados ou até mesmo por municípios vêm mostrando resultados positivos. O Estado de Minas Gerais vem sendo reconhecido pela sua melhoria no desempenho escolar, "o governo de Minas Gerais faz de sua educação básica a melhor do país - com base em avaliações de desempenho e programas de meritocracia" (FUCS, 2014). Um município do Estado do Ceará, Sobral, impressiona pela melhoria expressiva do desempenho escolar, o IDEB aplicado nos anos iniciais do Ensino Fundamental, que era de 4,0, em 2005, em 2013, subiu para 7,8, superando consideravelmente a meta estipulada pelo governo. A experiência de Sobral foi recomendada para todo o país como um exemplo de sucesso e boas práticas educacionais (INEP, 2005).

Dessa forma, nesse artigo pretende-se explorar a educação pública no Estado do Ceará, que embora tenha a peculiaridade de ter conseguido avanços nos 
resultados de educação nos últimos anos, ainda possui municípios com fatores socioeconômicos significativamente carentes. Portanto, este artigo tem como intuito mapear os municípios considerados benchmarking identificando seus fatores determinantes de sucesso e, por outro lado, para os municípios menos favorecidos, este artigo busca identificar os principais fatores de insucesso.

\section{Avaliação da educação no Brasil e avanços no Estado do Ceará}

A educação básica no Brasil é dividida em Ensino Fundamental e Ensino Médio. O Ensino Fundamental, por sua vez, é dividido em anos iniciais - do $1^{\circ}$ ao $4^{\circ}$ ano, e anos finais - do $5^{\circ}$ ao $9^{\circ}$ ano. O Ensino Médio engloba da $1^{\mathrm{a}}$ à $3^{\mathrm{a}}$ séries. Atualmente, o Estado do Ceará conta com 342.920 matrículas no Ensino Fundamental, divididos conforme mostra a Figura 1.

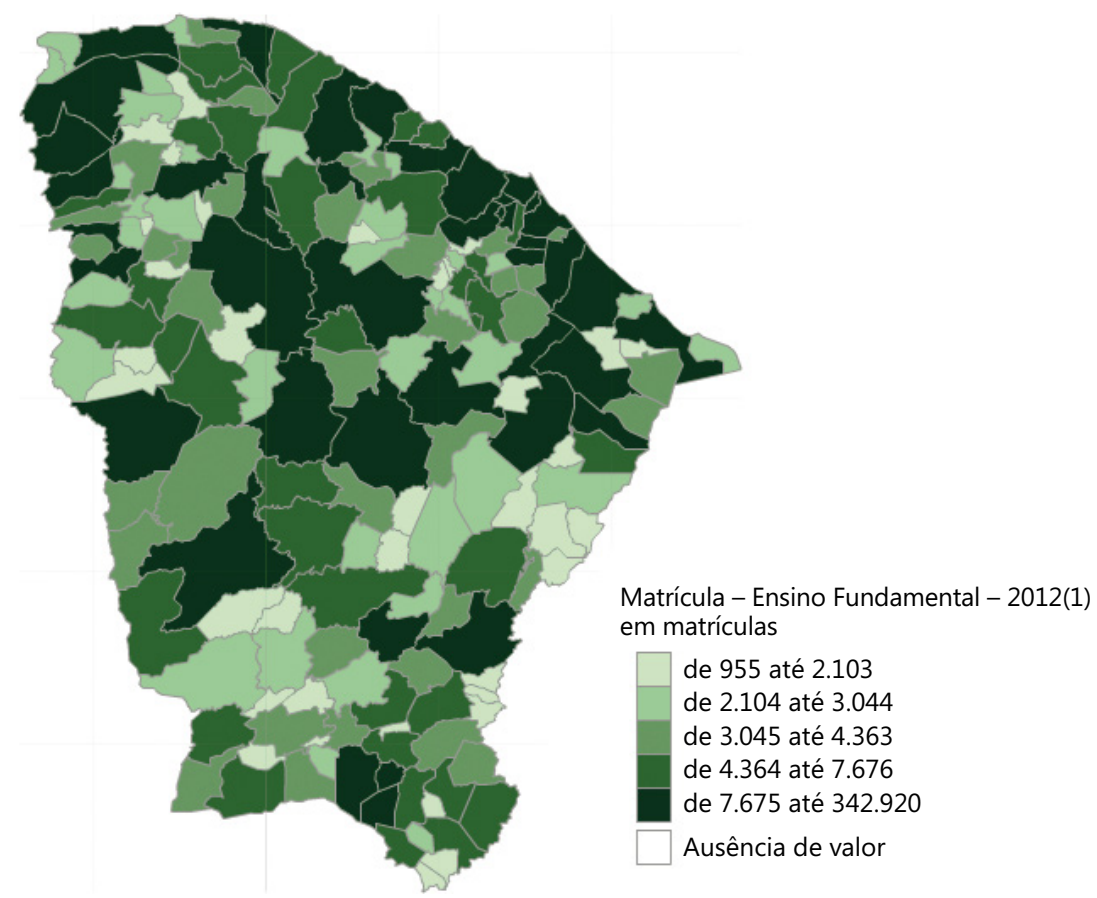

Fonte: (IBGE, 2012).

Figura 1. Número de matrículas no Ensino Fundamental no Estado do Ceará. 
Ao final de cada uma das etapas de ensino (anos iniciais, anos finais e Ensino Médio), a qualidade da educação é medida por meio do IDEB. O IDEB, realizado a cada dois anos, agrega dois conceitos referentes à qualidade da educação: o fluxo escolar e as médias de desempenho em exames padronizados aplicados pelo Instituto Nacional de Estudos e Pesquisas Educacionais Anísio Teixeira (INEP). O fluxo escolar é medido pela taxa de aprovação, ou rendimento escolar, coletado anualmente pelo INEP a partir do Censo Escolar. O desempenho escolar, por sua vez, é medido através de exames como a Prova Brasil e o Sistema de Avaliação da Educação Básica (SAEB). Com isso, pode-se verificar a taxa de alunos aprovados e que progridem dentro do sistema educacional e, além disto, se esses alunos conseguem ter um aprendizado satisfatório (Fonte: INEP, 2011).

O Ceará foi escolhido para este estudo pois vem apresentando evolução na qualidade da educação básica por meio de práticas não necessariamente inovadoras, mas bastante eficientes. Por exemplo, Sobral, uma cidade cearense com apenas 1,7\% da população do Estado apostou em um plano de gestão focado na erradicação do analfabetismo, na diminuição da evasão escolar, na valorização do professor e na meritocracia (BRETANHA, 2014)) com isso, o IDEB da cidade, que era de 4,0, em 2005 (anos iniciais), em 2013, alcançou a nota 7,8, superando consideravelmente a meta estipulada pelo governo. Como mencionado anteriormente, a experiência de Sobral foi recomendada para todo o país como um exemplo de sucesso e boas práticas educacionais (INEP, 2005). Algumas ações podem ser enumeradas no Estado do Ceará, por exemplo, em 2007, o Sistema Permanente de Avaliação da Educação Básica do Ceará (Spaece) foi redesenhado e estruturado em três grandes vertentes avaliativas: Avaliação da Alfabetização ( $2^{\circ}$ ano), Avaliação do Ensino Fundamental ( $5^{\circ}$ e $9^{\circ}$ anos) e Avaliação do Ensino Médio (1 $1^{\mathrm{a}}, 2^{\mathrm{a}} \mathrm{e}$ $3^{\mathrm{a}}$ séries) (LIMA, 2012); em 2009, o governo do Estado do Ceará instituiu o "Prêmio Escola Nota Dez", com o objetivo de fortalecer, valorizar e ampliar o trabalho que vem sendo empreendido pelas escolas em relação aos resultados de alfabetização (CEARÁ, 2015).

Também, outras ações são realizadas individualmente nos municípios, por exemplo, no município de Icapuí, CE,

[...] existem várias atividades ligadas à cultura e ao desporto, havendo 116 núcleos culturais com atividades semanais, um centro de memória da cultura popular, uma biblioteca pública, três bibliotecas escolares e três grupos de teatro de rua. Já com relação ao desporto, o município tem dois ginásios poliesportivos, cinco quadras 
esportivas e escolinhas de esporte. O município continua fornecendo transporte público e gratuito para as crianças de comunidades onde não há escolas, o que estimula as crianças a continuarem estudando (MARTINS; LOTTA, 2010).

Com isso, assim como Lima (2012), a Figura 2 mostra que, a partir de 2007, o Estado do Ceará revela uma tendência de melhoria no desempenho dos alunos tanto nos anos iniciais como nos finais, mas com intensidade acentuada nos anos iniciais.
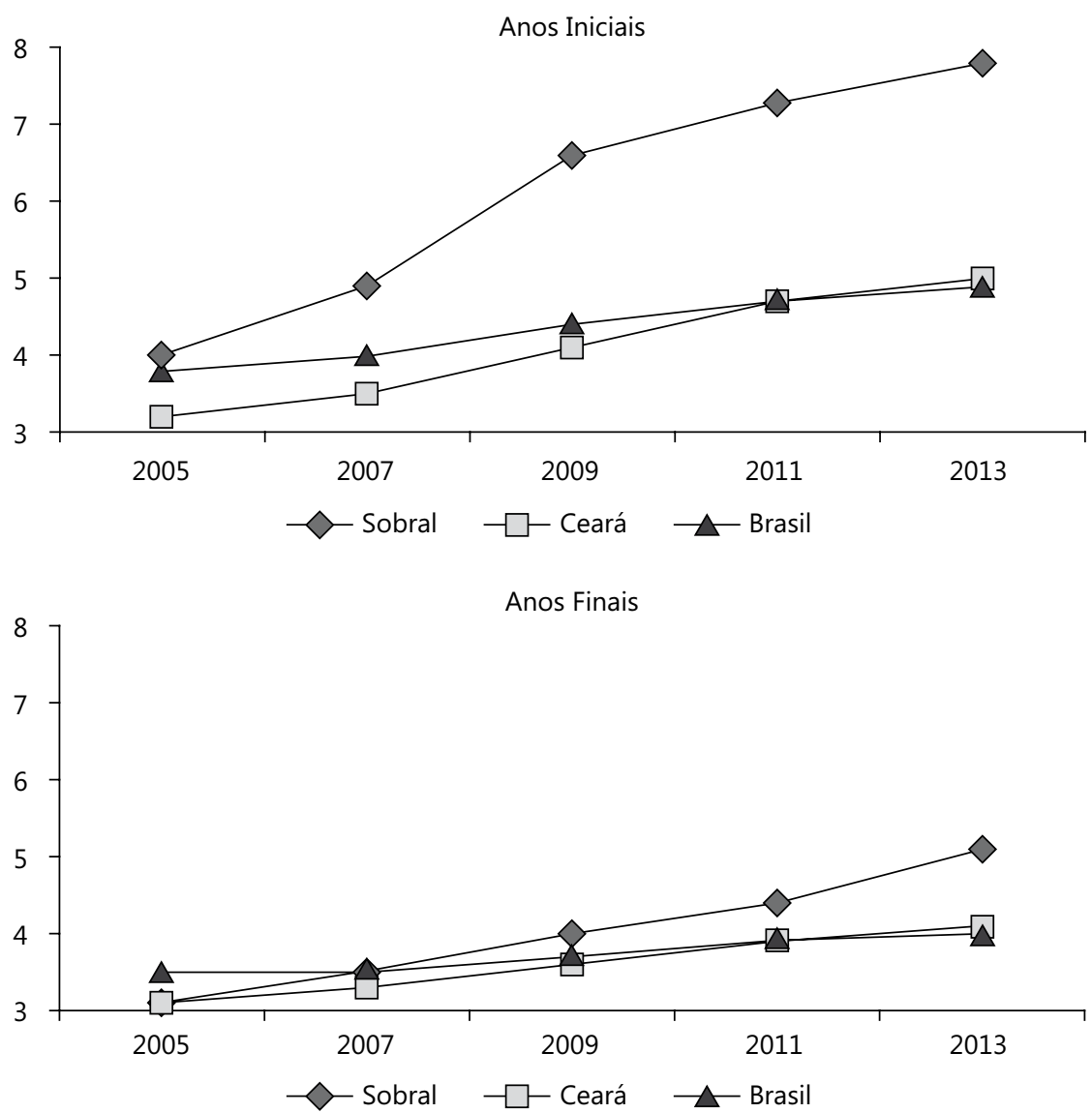

Fonte: Elaboração própria (2016).

Figura 2. Evolução das notas do IDEB para os anos iniciais e anos finais, numa escala de zero a dez. 
Segundo o Programa Alfabetização na Idade Certa (PAIC), criado em 2007, com o objetivo de prestar cooperação técnica e financeira aos municípios, com vista à melhoria dos resultados de aprendizagem, o nível de alfabetização do Estado do Ceará encontra-se em níveis desejável e suficiente (CEARÁ, 2016), mas para os anos seguintes, embora os resultados tenham melhorado significativamente para o Estado do Ceará, a Avaliação Nacional da Alfabetização (ANA), que mede o nível de proficiência em Escrita, Leitura e Matemática das crianças matriculadas no $3^{\circ}$ ano do ensino fundamental da rede pública, ainda mostra números abaixo do esperado em Escrita e, especialmente, em Matemática, conforme mostra a Tabela 1.

Além disso, percebe-se uma diminuição nas matrículas do Ensino Fundamental tanto no Estado do Ceará como no Brasil, percebe-se também uma tendência de diminuição bastante significativa em Sobral, chegando a 10\% de matrículas a menos entre 2013 e 2014 (Tabela 2). Uma justificativa para essa redução nas matrículas encontrada na literatura refere-se à diminuição da população, uma vez que as mulheres têm cada vez menos filhos.

\section{Metodologia}

A fim de analisar a eficiência educacional dos municípios do Estado do Ceará, utilizamos a análise envoltória de dados (Data Envelopment Analysis - DEA), uma metodologia de programação linear utilizada para medir a eficiência relativa de

Tabela 1. Nível de avaliação (em percentual) dos estudantes do estado do Ceará.

\begin{tabular}{lccc}
\hline Variável & Leitura & Escrita & Matemática \\
\hline Estudantes em nível adequado & 85,01 & 60,95 & 48,65 \\
Estudantes em nível inadequado & 14,99 & 39,06 & 51,34 \\
\hline
\end{tabular}

Fonte: INEP (2015).

Tabela 2. Evolução (em percentual) das matrículas no Ensino Fundamental.

\begin{tabular}{lcccccc}
\hline \multirow{2}{*}{$\begin{array}{l}\text { Matrículas } \\
\text { (\# estudantes) }\end{array}$} & \multicolumn{3}{c}{ Anos iniciais } & \multicolumn{3}{c}{ Anos finais } \\
\cline { 2 - 7 } & Sobral & Ceará & Brasil & Sobral & Ceará & Brasil \\
\hline $2010-2011$ & $-6,44$ & $-5,44$ & $-3,41$ & $-2,86$ & $-4,64$ & $-2,51$ \\
$2011-2012$ & $-3,01$ & $-4,79$ & $-3,44$ & $-6,77$ & $-4,75$ & $-2,77$ \\
$2012-2013$ & $-1,83$ & $-5,24$ & $-2,57$ & $-6,77$ & $-2,77$ & $-3,30$ \\
$2013-2014$ & $-2,67$ & $-3,16$ & $-1,23$ & $-10,00$ & $-2,59$ & $-4,77$ \\
\hline
\end{tabular}

Fonte: QEdu (2015). 
unidades produtivas semelhantes (Decision Making Units - DMUs) com múltiplos insumos e produtos (Figura 3). As unidades produtivas a serem analisadas devem usar os mesmos recursos e produzir os mesmos produtos, variando apenas na quantidade a ser utilizada e produzida, gerando, então, a eficiência relativa de cada unidade produtiva, ou seja, a unidade com melhor relação produtos/recursos obtém a melhor eficiência relativa.

Matematicamente, considerando $n$ variáveis de inputs (recursos) e $s$ variáveis de outputs (produtos), a metodologia DEA calcula a eficiência das $m$ DMUs $(u=1, \ldots, m)$ de forma a determinar os pesos $(x$ e $y)$, tal que maximize a razão da função objetivo abaixo.

$$
\operatorname{Max}_{u}=\frac{\sum_{j=1}^{s} y_{j} O_{j u}}{\sum_{i=1}^{n} x_{i} I_{i u}}
$$

Sujeito a: $\quad \frac{\sum_{j=1}^{s} y_{j} O_{j k}}{\sum_{i=1}^{n} x_{i} I_{i k}} \leq 1 ; k=1, \ldots, m$

$y_{j} \geq 0 ; j=1, \ldots, s$

$x_{i} \geq 0 ; i=1, \ldots, n \quad$,

A técnica DEA vem sendo bastante aplicada no setor de educação, embora em maior quantidade no Ensino Superior (AHN, et al., 1988; BARBOSA; WILHEM, 2009; FAÇANHA et al., 1997; HU et al., 2009; JOHNES; JOHNES, 1993; MARINHO et al., 1997; entre outros). Na educação básica a DEA ainda é pouco explorada, Pedroso et al. (2009) usam a DEA para a avaliação da gestão do Programa Bolsa Família nas capitais brasileiras, identificando as capitais detentoras de "boas práticas" em gestão do programa. Barbosa e Wilhem (2009) analisaram o desempenho das escolas de Ensino Fundamental e Médio do Núcleo Regional de Educação de Paranavaí - Paraná. O estudo mostra que ajustes na gestão destas escolas poderiam melhorar significativamente seus resultados.

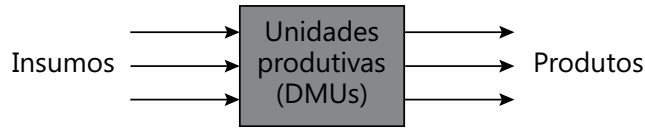

Fonte: Elaboração própria (2016).

Figura 3. Representação da técnica DEA. 
A técnica DEA pode ser usada tanto com retorno constante de escala (modelo CRS - Constant Returns to Scale), ou seja, um aumento nos insumos gera um aumento proporcional nos produtos (CHARNES et al., 1978), ou com retorno variável de escala (modelo VRS - Variable Returns to Scale), que considera situações de eficiência de produção com variação de escala e não assume proporcionalidade entre insumos e produtos (BANKER et al., 1984). Em geral, recomenda-se usar o modelo CRS para DMUs com portes semelhantes, caso contrário, pode haver uma distorção dos resultados gerando mais eficiência para DMUs maiores.

Além disso, também se deve escolher a orientação da otimização: orientação a insumos (quando se deseja minimizar os insumos, mantendo os valores dos produtos constantes) ou orientação a produtos (quando se deseja maximizar os resultados mantendo o nível de recursos constante).

Para este estudo, o modelo levou em consideração 172 municípios do Estado do Ceará como as DMUs, e oito variáveis relacionadas a cada um dos municípios (Quadro); uma variável de input: o gasto per capita, e sete variáveis de output referentes aos seguintes fatores:

- Professores: Prof_sup e Prof_licenc_area

- Desempenho escolar: IDEB-AI e IDEB-AF

- Socioeconômico: Escolas_esgoto_san, Mães_edu e IDH-R.

Dessa forma, o modelo assume que quanto maior o gasto per capita (input), maiores os outputs, ou seja, maior o número de professores com Ensino Superior, maior o número de professores com Licenciatura na área que atuam, melhores notas IDEB-AI e IDEB-AF, maior o número de escolas com acesso a esgoto sanitário, melhor a educação das mães e gerando, também, maior renda per capita do município.

Usaremos o modelo VRS, orientado a output, ou seja, desejamos saber quais municípios geram melhores outputs com o gasto per capita (input) atual.

O Quadro a seguir mostra a descrição das variáveis e suas respectivas fontes.

A variável Gasto per capita foi calculada como a divisão do gasto total em educação no Estado do Ceará dividido pelo número de matrículas no Ensino Fundamental para cada município. 
Quadro. Descrição e fonte das variáveis.

\begin{tabular}{|c|c|c|}
\hline Variável & Descrição & Fonte \\
\hline $\begin{array}{l}\text { Gasto per } \\
\text { capita }\end{array}$ & $\begin{array}{l}\text { Gasto por aluno no Ensino } \\
\text { Fundamental (2010). }\end{array}$ & $\begin{array}{l}\text { Fundo Nacional de } \\
\text { Desenvolvimento da Educação, } \\
\text { Sistema SIOPE, } 2010 .\end{array}$ \\
\hline Prof_sup & $\begin{array}{l}\text { Percentual de professores do Ensino } \\
\text { Fundamental com Ensino Superior } \\
\text { (2010). }\end{array}$ & $\begin{array}{l}\text { Observatório do Plano } \\
\text { Nacional de Educação (www. } \\
\text { observatoriodopne.org.br) }\end{array}$ \\
\hline $\begin{array}{l}\text { Prof_licenc_ } \\
\text { area }\end{array}$ & $\begin{array}{l}\text { Percentual de professores dos anos } \\
\text { finais do Ensino Fundamental que tem } \\
\text { Licenciatura na área em que atuam } \\
\text { (2010). }\end{array}$ & $\begin{array}{l}\text { Observatório do Plano } \\
\text { Nacional de Educação (www. } \\
\text { observatoriodopne.org.br) }\end{array}$ \\
\hline IDEB-AI & $\begin{array}{l}\text { Nota padronizada (IDEB) para anos } \\
\text { iniciais do Ensino Fundamental da rede } \\
\text { pública (2011). }\end{array}$ & www.qedu.org.br \\
\hline IDEB-AF & $\begin{array}{l}\text { Nota padronizada (IDEB) para anos } \\
\text { finais do Ensino Fundamental da rede } \\
\text { pública (2011). }\end{array}$ & www.qedu.org.br \\
\hline $\begin{array}{l}\text { Escolas__ } \\
\text { esgoto_san }\end{array}$ & $\begin{array}{l}\text { Porcentagem de escolas da rede } \\
\text { pública do Ensino Fundamental com } \\
\text { acesso a esgoto sanitário (2010). }\end{array}$ & $\begin{array}{l}\text { Observatório do Plano } \\
\text { Nacional de Educação (www. } \\
\text { observatoriodopne.org.br) }\end{array}$ \\
\hline Mães_edu & $\begin{array}{c}100 \text { - percentual de mães chefes de } \\
\text { família sem fundamental completo e } \\
\text { com filhos menores de } 15 \text { anos (2010). }\end{array}$ & $\begin{array}{l}\text { Observatório do Plano } \\
\text { Nacional de Educação (www. } \\
\text { observatoriodopne.org.br) }\end{array}$ \\
\hline IDH-R & $\begin{array}{l}\text { Índice de Desenvolvimento Humano } \\
\text { relativo à renda (2010). }\end{array}$ & $\begin{array}{l}\text { Observatório do Plano } \\
\text { Nacional de Educação (www. } \\
\text { observatoriodopne.org.br) }\end{array}$ \\
\hline
\end{tabular}

Fonte: Elaboração própria (2016).

\section{Dados}

O Estado do Ceará, localizado no Nordeste do Brasil, com uma população em torno de 8,43 milhões, conta com 181 municípios, sendo Fortaleza a sua capital. Neste estudo, utilizamos os dados de 172 municípios devido à falta de dados para os demais. A Tabela 3 a seguir mostra a estatística descritiva das variáveis utilizadas no modelo.

Embora a Tabela 3 mostre que o máximo da variável Escolas_esgoto_san é de $91,5 \%$, vale mostrar que apenas dois municípios possuem essa variável com alto valor, 4 municípios possuem entre $51 \%$ e $70 \%$ das escolas com acesso a esgoto sanitário, e 166 municípios possuem menos de 51\% das escolas com acesso a esgoto sanitário, conforme mostra a Tabela 4. 
Tabela 3. Estatística descritiva.

\begin{tabular}{lcccccccc}
\hline Variável & $\begin{array}{c}\text { Gasto } \\
\text { per } \\
\text { capita }\end{array}$ & $\begin{array}{c}\text { Prof_- } \\
\text { sup }\end{array}$ & $\begin{array}{c}\text { Prof_- } \\
\text { licenc_ } \\
\text { area }\end{array}$ & IDEB-Al & IDEB-AF & $\begin{array}{c}\text { Escolas_ } \\
\text { esgoto__ } \\
\text { san }\end{array}$ & $\begin{array}{c}\text { Mães_ } \\
\text { edu }\end{array}$ & IDH-R \\
\hline Mínimo & $1.868,90$ & 23,9 & 0 & 3,3 & 2,9 & 0 & 71 & 0,49 \\
Máximo & $5.028,70$ & 100 & 37,9 & 7,5 & 5,4 & 91,5 & 97,6 & 0,75 \\
Média & $3.023,10$ & 65 & 9,8 & 4,8 & 3,9 & 11,5 & 86,7 & 0,56 \\
Desv pad & 501,7 & 15,7 & 7 & 0,7 & 0,4 & 14,8 & 4,4 & 0,04 \\
\hline
\end{tabular}

Fonte: Elaboração própria (2016).

Tabela 4. Quantidade de municípios com percentual de escolas com acesso a esgoto sanitário.

\begin{tabular}{lc}
\hline Percentual de escolas & $\begin{array}{c}\text { Quantidade de municípios com escolas com acesso a } \\
\text { esgoto sanitário }\end{array}$ \\
\hline $91-100 \%$ & 1 \\
$71-90 \%$ & 1 \\
$51-70 \%$ & 4 \\
Abaixo de 51\% & 166 \\
\hline
\end{tabular}

Fonte: Elaboração própria (2016).

\section{Resultados empíricos}

Aplicamos a análise envoltória de dados utilizando o software Frontier Analyst 4. Utilizamos o modelo VRS orientado a output, a fim de determinar quais municípios conseguem obter melhores resultados e melhores condições de professores, desempenho e fatores socioeconômicos relacionados à educação, com os gastos per capita atuais, ou seja, quais os municípios mais eficientes.

Neste artigo, vamos considerar municípios benchmarking, aqueles que têm $100 \%$ de eficiência e são referência para, pelo menos, 10 outros municípios, pois municípios que são referência para poucas DMUs (ou, até mesmo, referência somente para ele próprio) podem ter sido escolhidos como eficientes por apenas uma variável se destacar e não por um conjunto de variáveis com bom desempenho. Os resultados apontam 18 municípios considerados benchmarking, ou seja, esses municípios obtiveram $100 \%$ de eficiência (relativa) e são referência para, pelo menos, outros 10 municípios. A Figura 4 mostra a localização dos municípios considerados benchmarking, o nome, e entre parênteses, o número de municípios 
para os quais este benchmarking é referência; por exemplo, o município de Lavras da Mangabeira é referência para outros 86 municípios, incluindo ele próprio.

É claro que, mesmo sendo referência para no mínimo outros 10 municípios, pode acontecer de um município ter alguma variável com um valor baixo, mas que pode ser compensado por outras variáveis terem valores bastante superiores e, ainda assim, o município pode ser considerado um benchmarking. É o próprio caso de Lavras de Mangabeira, que possui apenas 6,5\% das escolas com acesso a esgoto sanitário, mas tem um gasto per capita bastante baixo. Entretanto, vale lembrar a Tabela 4, que mostra que $96,5 \%$ dos municípios têm menos que 50\% das escolas com acesso a esgoto sanitário.

Na Figura 4, percebe-se claramente uma aglomeração dos municípios benchmarking em três regiões, uma próxima a Sobral, outra próxima a Fortaleza e outra próxima a Juazeiro do Norte. Essas três localizações, além de serem mais populosas, abrigam exatamente nessas localizações os três campi do curso de Medicina da Universidade Federal do Ceará, localizados em Fortaleza, Sobral e Barbalha (situada próxima a Juazeiro do Norte), além da Universidade Federal do Cariri, situada em Juazeiro do Norte (pontos em formato de estrela na Figura 4).

Este resultado corrobora com os resultados obtidos por Barros e Freire (2011), que analisaram ações de responsabilidade social do Curso de Medicina da Universidade Federal do Ceará, em Sobral, e pôde-se perceber que o curso desenvolve ações sociais que oferecem respostas positivas à comunidade em seu entorno, embora ainda se tenha muito a ser melhorado no sentido de responsabilidade social.

A Figura 5 mostra:

1. Para cada município benchmarking os valores das variáveis;

2. A média das variáveis dos municípios benchmarking;

3. A média das variáveis dos demais municípios (excluindo os municípios benchmarking) com eficiência entre 91 e $100 \%$ e, finalmente,

4. A média das variáveis dos municípios com eficiência entre 81 e $90 \%$.

Por exemplo, na Figura 5, o gráfico Prof_sup mostra: 
1. Para cada município, o percentual de professores com nível superior (por exemplo, o município Lavras de Mangabeira possui $71 \%$ dos professores com Ensino Superior);

2. Que, na média, os municípios benchmarking têm $75 \%$ dos professores com nível superior;

3. Que, na média, os municípios com eficiência entre 91 e 100\% (excluindo os municípios benchmarking) têm $65 \%$ dos professores com nível superior e

4. Que, na média, os municípios com eficiência entre 81 e $90 \%$ têm $53 \%$ dos professores com Ensino Superior.

Claro que a média dos municípios benchmarking é sempre maior do que a média dos municípios com eficiência entre 91 e $100 \%$ e maior que a média dos municípios com eficiência entre 81 e 90\%.

Nota-se que, mesmo dentre os municípios considerados benchmarking, existe uma variação nos valores das variáveis. Estes municípios não são necessariamente os que possuem melhores valores em todas as variáveis consideradas no modelo. Por exemplo, o município Deputado Irapuan Pinheiro possui um valor consideravelmente baixo de professores com Ensino Superior (Prof_sup), mas compensado pelos valores acima da média tanto no IDEB-AI como no IDEB-AF. O município de Pacajus, que possui o menor valor do gasto per capita, tem os valores das demais variáveis muito próximos à média geral (alguns até abaixo), ou seja, o baixo valor do gasto per capita fez com que este município seja considerado um benchmarking, pois consegue resultados (outputs) próximos à média com um gasto per capita significativamente menor. É importante notar também que, mesmo dentre os municípios considerados benchmarking, a média de escolas com acesso a esgoto sanitário é bastante baixa, de 19,8\%.

A metodologia DEA fornece os pesos atribuídos a cada variável para que cada município tenha a melhor eficiência possível, ou seja, os maiores pesos são considerados os fatores de eficiência daquele município e, consequentemente, os menores pesos são as deficiências do mesmo. A Figura 6 mostra os pesos dados a cada variável no modelo DEA, ou seja, as variáveis que mais influenciaram na eficiência de cada município, o que denominamos de Determinantes de Eficiência. Na Figura 6, analisamos os municípios considerados benchmarking, os municípios com eficiência entre $91 \mathrm{e}$ 100\% (excluindo os municípios considerados benchmarking), e os municípios com eficiência entre 81 e $90 \%$. Fica claro que, nos três intervalos de análise, os maiores 


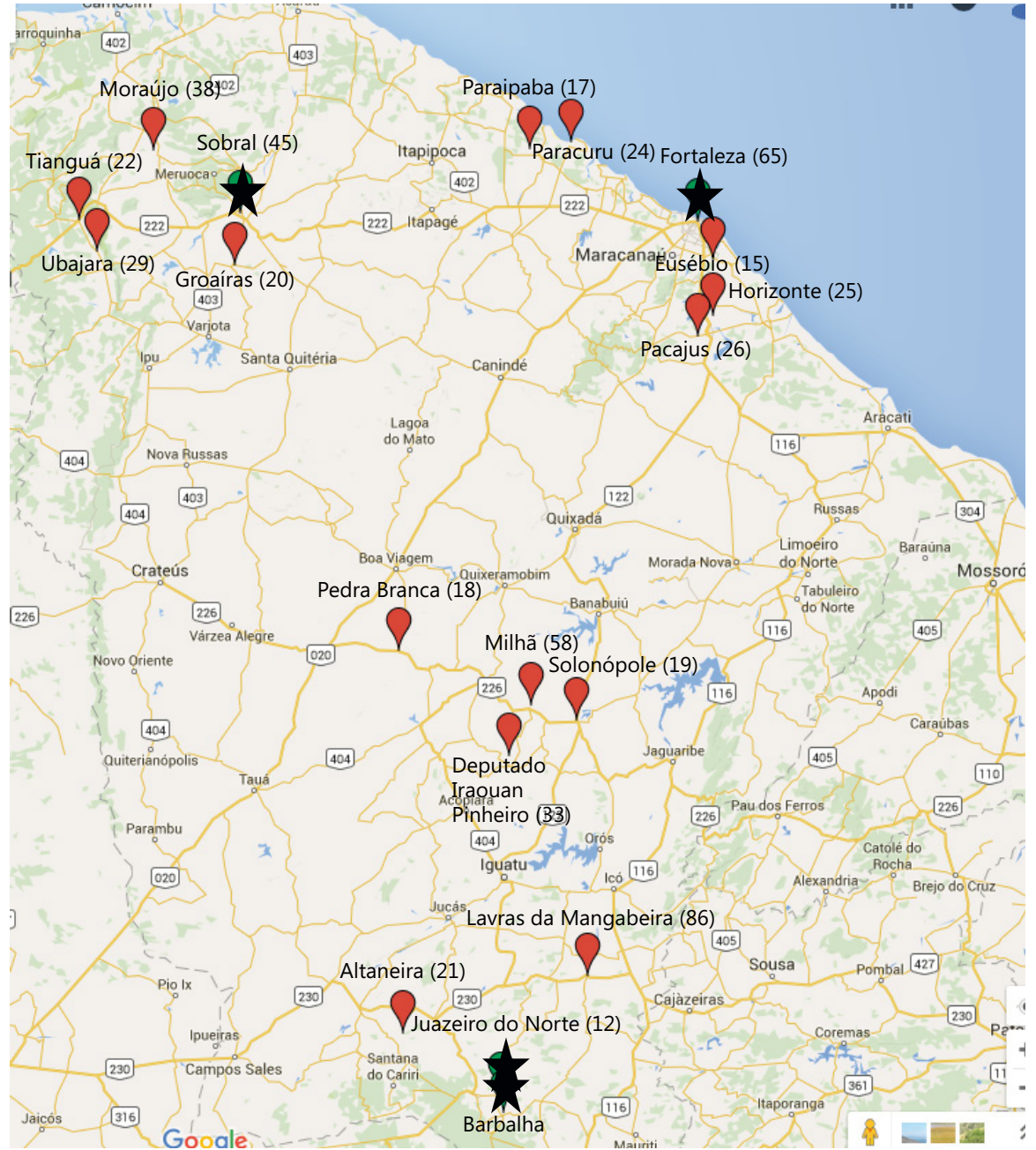

Fonte: Elaboração própria (2016).

Figura 4. Mapa dos municípios cearenses considerados benchmarking.

pesos foram dados a variáveis referentes ao gasto e educação das mães, e o fator renda se mostrou também um determinante de eficiência principalmente para os municípios menos eficientes, as demais variáveis receberam um peso significativamente menor.

Entretanto, dentre as variáveis que receberam menor peso, duas variáveis se sobressaem, Prof_licenc_area e Escolas_esgoto_san são as variáveis que receberam 
Prof_sup

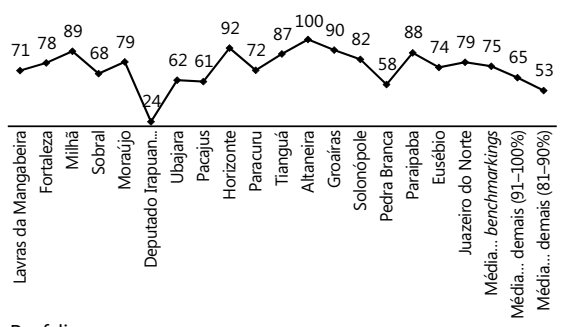

Prof_licenc_area

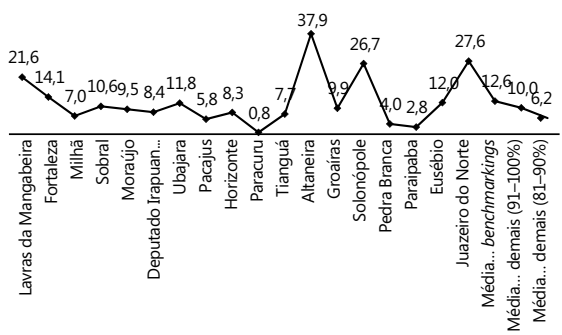

Escolas_esgoto_san

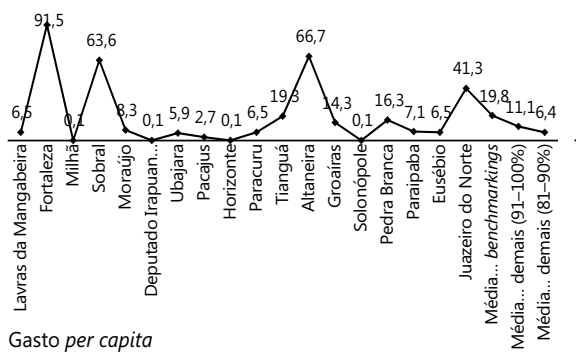

(2.50)

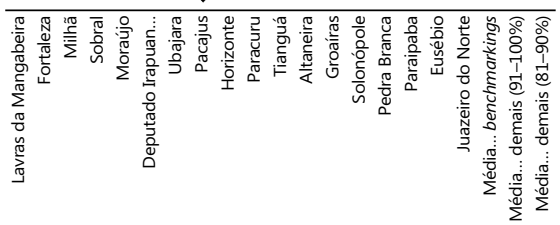

IDEB -AI

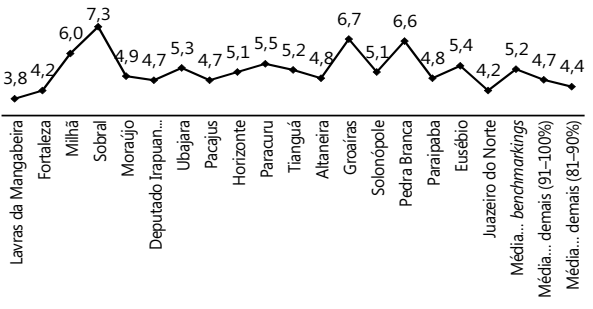

IDEB -AF

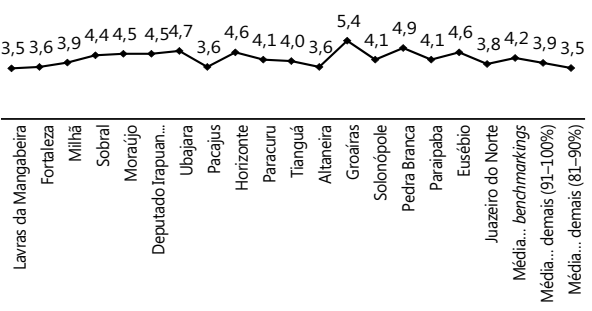

Mães_edu
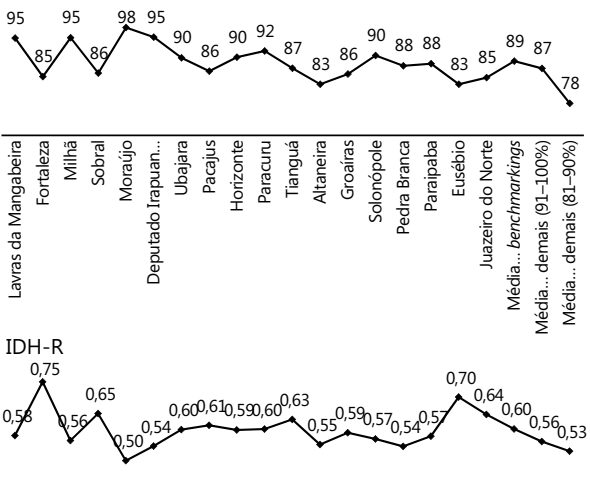

Fonte: Elaboração própria (2016).

Figura 5. Panorama dos municípios por intervalo de eficiência.

pesos praticamente nulos, principalmente esta última, o que corrobora com Campos (2000) que relata "[...] no caso do Brasil, os indicadores de qualidade utilizados pelos levantamentos realizados revelam que a escola frequentada pela maioria 
Determinantes de eficiência

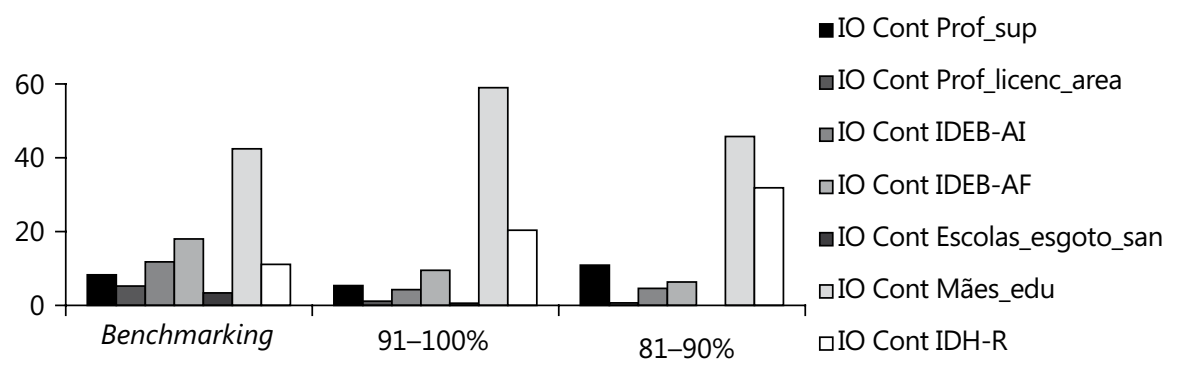

Fonte: Elaboração própria (2016).

Figura 6. Média dos pesos atribuídos às variáveis.

da população ainda não apresenta condições mínimas para a aprendizagem de conhecimentos básicos".

\section{Considerações Finais}

Este estudo teve como objetivo identificar os determinantes de eficiência educacional dos municípios do Estado do Ceará, que vem mostrando melhoras expressivas no seu desempenho escolar, principalmente nos anos iniciais do Ensino Fundamental.

Um modelo de eficiência educacional foi formulado e, usando a metodologia DEA, 172 municípios foram analisados. Para isso, foram utilizadas oito variáveis, um input, o gasto per capita e sete outputs relacionados a professores, qualidade da educação e fatores socioeconômicos que influenciam diretamente a educação (como renda, educação materna e acesso a esgoto sanitário).

Como resultados principais, pode-se destacar que os municípios benchmarkings estão aglomerados geograficamente em três clusters, justamente onde se situam os campi do curso de Medicina da Universidade Federal do Ceará, os quais impulsionam a qualidade da educação em suas regiões (BARROS; FREIRE, 2011). Os resultados também levam aos determinantes de eficiência, ou seja, às variáveis que mais impulsionaram a eficiência dos municípios benchmarkings, que são: gasto per capita, educação das mães e renda. Por outro lado, o determinante de ineficiência, ou seja, as variáveis que receberam os menores pesos (em muitos casos, até peso nulo), foi o número de escolas com acesso a esgoto sanitário e o número de professores com Licenciatura na área em que atuam, ou seja, essas podem ser consideradas as variáveis com maior potencial de melhorias. 
Finalmente, este artigo reafirma que a educação não pode ser vista como um pilar isolado, os fatores socioeconômicos, como saneamento básico, renda e outros fatores que influenciam a qualidade de vida mínima possuem uma influência tão ou até mais forte sobre a educação do que fatores diretamente ligados a ela, como escolas e professores. 


\title{
Analysis of the determinants of educational efficiency of the state of Ceará
}

\begin{abstract}
The state of Ceará has shown significant improvements in the education sector since 2007, especially in the early years of elementary school which has already surpassed the educational assessment targets set by the government. Thus this article seeks to identify the determinants of efficiency that led this state to improve its educational performance, considering variables directly linked to education, as well as socioeconomic variables, which have great influence in this sector. The results clearly show the existence of clusters of municipalities considered benchmarking that geographically coincide with the locations of the three campuses of the medical school of the Federal University of Ceará. The variables, income, per capita expenditure and maternal education were shown as determinants of efficiency of Ceará municipalities, but the variable related to access to sanitary sewer showed to be a strong determinant of inefficiency, especially for the poor municipalities.
\end{abstract}

Keywords: Basic education. Teaching quality. Efficiency.

\section{El análisis de los determinantes de la eficiencia educativa del estado de Ceará}

\section{Resumen}

El estado de Ceará ha demostrado mejoras significativas en el sector de la educación desde 2007, especialmente en los primeros años de la escuela primaria, que ya ha superado los objetivos de evaluación educativa establecidos por el gobierno. Así, este artículo busca identificar los factores determinantes de la eficiencia que llevaron a este estado a mejorar su rendimiento educativo, teniendo en cuenta las variables directamente vinculadas a la educación, así como las variables socioeconómicas, que tienen gran influencia en este sector. Los resultados muestran claramente la existencia de agrupaciones geográficas de municipios considerados con mejores calificaciones y que coinciden con las instalaciones de los tres campus de la facultad de medicina de la Universidad Federal de Ceará. Las variables, los ingresos per cápita, el gasto per cápita y la educación materna se muestran como factores determinantes de la eficiencia de los municipios de Ceará; por otro lado, la variable relacionada con el acceso a alcantarillado se muestra como un fuerte determinante de la ineficiencia, especialmente para los municipios pobres.

Palabras clave: Educación básica. La calidad de la enseñanza. La eficiencia. 


\section{Referências}

AHN, T.; CHARNES, A.; COOPER, W. Some statistical and DEA evaluations of relative efficiencies of public and private institutions of higher learning. Socio-Economic Planning Sciences, v. 22, n. 6, p. 259-69, 1988. http://doi.org/10.1016/0038-0121(88)90008-0

BANKER, R. D.; CHARNES, A.; COOPER, W. W. Some models for estimating technical and scale efficiencies in Data Envelopment Analysis. Management Science, v. 30, n. 9, p. 1078-92, 1984. http://doi.org/10.1287/mnsc.30.9.1078

BARBOSA, S. G.; WILHEM, V. E. Avaliação do desempenho das escolas públicas por meio de Data Envelopment Analysis. Acta Scientiarum. Technology, v. 31, n. 1, p. 71-9, 2009. http://doi.org/10.4025/actascitechnol.v31i1.1547

BARROS, C. M. P., FREIRE, J. C. A responsabilidade social universitária na perspectiva do Sinaes: um estudo de caso no Curso de Medicina da Universidade Federal do Ceará - Campus de Sobral. Ensaio: Avaliação e Políticas Públicas em Educação, v. 19, n. 73, p. 891-920, 2011. http://doi.org/10.1590/S0104-40362011000500009

BRETANHA, B. Sobral vira modelo nacional de gestão. Estadão, 25 abr. 2014. Educação. Disponível em: <http://educacao.estadao.com.br/noticias/geral,sobralvira-modelo-nacional-de-gestao-imp-,1158465>. Acesso em: 19 abr. 2016.

CALDERÓN, A. I., RAQUEL, B. M. G.; CABRAL, E. S. O Prêmio Escola nota 10: meritocracia e cooperação para a melhoria do desempenho escolar. Ensaio: Avaliação e Políticas Públicas em Educação, v. 23, n. 87, p. 517-40, 2015. http://doi.org/10.1590/S0104-40362015000100021

CAMPOS, M. M. A qualidade da educação em debate. São Paulo: Campanha Nacional pelo Direito à Educação, 2000. (Cadernos do Observatório, v. 2).

CEARÁ. Governo do Estado. Secretaria da Educação. MAISPAIC. Mapas dos resultados. Fortaleza, 2016. Disponível em: http://www.paic.seduc.ce.gov.br/ index.php/resultados/mapas-dos-resultados. Acesso em: 12 dez. 2016.

CEARÁ. Governo do Estado. Secretaria da Educação. MAISPAIC. Prêmio Escola Nota 10. Fortaleza, 2015. Disponível em: <http://www.paic.seduc. ce.gov.br/index.php/o-paic/premio-escola-nota-10>. Acesso em: 19 abr. 2016. 
CHARNES, A.; COOPER, W. W.; RHODES, E. Measuring efficiency of decision making units. European Journal of Operational Research, v. 2, n. 6, p. 429-44, 1979. http://doi.org/10.1016/0377-2217(78)90138-8

CLÍMACO, N. L. Ações da Capes para a formação e a valorização dos professores da educação básica do Brasil. Revista de Pós-Graduação, RBPG, v. 9, n. 16, 2012.

FUCS, J. Como Minas Gerais conseguiu a melhor educação básica do país. Época, 6 maio 2014. Disponível em: <http://epoca.globo.com/tempo/ noticia/2014/05/como-bminas-geraisb-conseguiu-melhor-educacao-basica-dopais.html>. Acesso em: 19 abr. 2016.

FAÇANHA, L.; REZENDE, M.; MARINHO, A. Brazilian federal universities: relative efficiency evaluation and data envelopment analysis. Revista Brasileira de Economia, v. 51, n. 4, p. 489-508, 1997.

HU, Y.; ZHANG, Z.; LIANG, W. Efficiency of primary schools in Beijing, China: an evaluation by data envelopment analysis. International Journal of Educational Management, v. 23, n. 1, p. 34-50, 2009. http://doi.org/10.1108/09513540910926411

INSTITUTO BRASILEIRO DE GEOGRAFIA E ESTATÍSTICA - IBGE. Cidades. Ceará e Fortaleza: ensino: matrículas, docentes e rede escolar: matrícula ensino fundamental - 2012. Rio de Janeiro, 2012. Disponível em: $<$ http://www.cidades.ibge.gov.br/cartograma/mapa.php?lang=\&coduf=23\&co $\mathrm{dmun}=230440 \&$ idtema $=117 \& \mathrm{codv}=\mathrm{V} 01 \&$ search $=$ ceara|fortaleza|sintese-dasinformacoes-2012>. Acesso em: 19 abr. 2016.

INSTITUTO NACIONAL DE ESTUDOS E PESQUISAS EDUCACIONAIS ANÍSIO TEIXEIRA - INEP. Avaliação nacional da alfabetização 2014. Brasília, DF: INEP, 2015. Disponível em: $<$ http://portal.mec.gov.br/index.php?option=com docman\&view=download\&alias=21091-apresentacao-ana-15-pdf\&category_ slug=setembro-2015-pdf\&Itemid=30192>. Acesso em: 12 dez. 2016.

. Como o Ideb é calculado. Brasília, DF: INEP, 2011. Disponível em: $<$ http://portal.inep.gov.br/web/portal-ideb/como-o-ideb-e-calculado $>$. Acesso em: 19 abr. 2016.

. Vencendo o desafio da aprendizagem nas séries iniciais: a experiência de Sobral/CE. Brasília, DF: INEP, 2005.

JOHNES, G.; JOHNES, J. Measuring the research performance of UK Economics Departments: an application of data envelopment analysis. Oxford Economic Papers, New Series, v. 45, n. 2, p. 332-47, 1993. 
LIMA, A. Ciclo de avaliação da educação básica do Ceará: principais resultados. Estudos em Avaliação Educacional, v. 23, n. 53, p. 38-58, set./dez. 2012. http://doi.org/10.18222/eae235320121914

MARINHO, A.; RESENDE, M.; FAÇANHA, L. Brazilian federal universities: relative efficiency evaluation and data envelopment analysis. Revista Brasileira de Economia, v. 51, n. 4, p. 489-508, 1997.

MARTINS, R. D., LOTTA, G. S. Capital social e redes sociais como alternativa para análise de políticas públicas de educação: o caso de Icapuí-CE. Ensaio: Avaliação e Políticas Públicas em Educação, v. 18, n. 69, p. 846-60, 2010. http://doi.org/10.1590/S0104-40362010000400010

PEDROSO, M. M.; CALMON, P. C. D. P.; BANDEIRA, L. F. O uso de Análise Envoltória de Dados para avaliação da gestão do Programa Bolsa-Famíla. Comunicação em Ciências da Saúde, v. 20, n. 1, p. 37-44, 2009.

QEdu. Brasil. Ceará. Matrículas e infraestrutura. 2015. Disponível em: $<$ http://www.qedu.org.br/estado/106-ceara/censo-escolar?year=2014\&locali zation $=0 \&$ dependence $=0 \&$ education_stage $=0 \&$ item $=$ matriculas-no-ensinofundamental>. Acesso em: $12 \mathrm{dez} .2016$.

\section{Informações da autora}

Maria Cristina Gramani: Doutora em Engenharia pela Universidade Estadual de Campinas - Unicamp. Professora Associada do Insper - Instituto de Ensino e Pesquisa. Contato: mariacng@insper.edu.br 